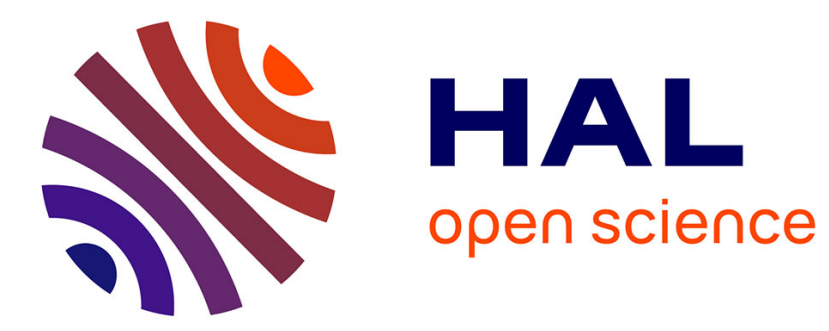

\title{
Amélioration du rendement de pompage optique dans les lasers à iode
}

\author{
M. Bédu, M. Jeanjean
}

\section{To cite this version:}

M. Bédu, M. Jeanjean. Amélioration du rendement de pompage optique dans les lasers à iode. Revue de Physique Appliquée, 1983, 18 (3), pp.191-201. 10.1051/rphysap:01983001803019100 . jpa00245084

\section{HAL Id: jpa-00245084 https://hal.science/jpa-00245084}

Submitted on 1 Jan 1983

HAL is a multi-disciplinary open access archive for the deposit and dissemination of scientific research documents, whether they are published or not. The documents may come from teaching and research institutions in France or abroad, or from public or private research centers.
L'archive ouverte pluridisciplinaire HAL, est destinée au dépôt et à la diffusion de documents scientifiques de niveau recherche, publiés ou non, émanant des établissements d'enseignement et de recherche français ou étrangers, des laboratoires publics ou privés. 


\title{
Amélioration du rendement de pompage optique dans les lasers à iode
}

\author{
M. Bédu et M. Jeanjean \\ Commissariat à l'Energie Atomique, Centre d'Etudes de Limeil, B.P. nº 27, 94190 Villeneuve Saint Georges, France
}

(Reçu le $1^{\mathrm{er}}$ juin 1981, révisé le ler décembre 1982, accepté le 14 décembre 1982)

\begin{abstract}
Résumé. - Lors d'un travail expérimental sur des flashes au xénon basse pression (10 à 80 torrs), employés pour réaliser le "pompage optique " des lasers à iode, nous avons étudié un procédé qui permet d'augmenter la production du rayonnement UV et donc le rendement de pompage du laser (rapport entre l'énergie stockée par inversion de population dans le milieu amplificateur et l'énergie de pompage).
\end{abstract}

Abstract. - Within an experimental work on low pressure xenon flash-lamps (10 to 80 torrs) used for optical pumping of iodine lasers, we have studied a process improving UV production, therefore increasing the laser pumping efficiency (ratio between stored energy by inversion population in amplifying medium and pumping energy).

1. Introduction. - Le laser à iode, utilisant comme milieu actif l'iode atomique excité provenant de la photodissociation de composés iodés tels que le $\mathrm{C}_{3} \mathrm{~F}_{7} \mathrm{I}$ [1], est un candidat très intéressant en matière de source laser pour les expériences de fusion par confinement inertiel $[2,3]$.

Un des points fondamentaux est le rendement de cette source laser et donc l'efficacité du pompage optique utilisé pour obtenir la photodissociation, dans la bande spectrale d'absorption utile (voir Fig. 1), du composé iodé. Deux techniques sont actuellement utilisées pour obtenir ce rayonnement dans le proche ultraviolet : les fils explosés [4] et les flashes au xénon [5-7]. Cette dernière, plus largement utilisée, et bien

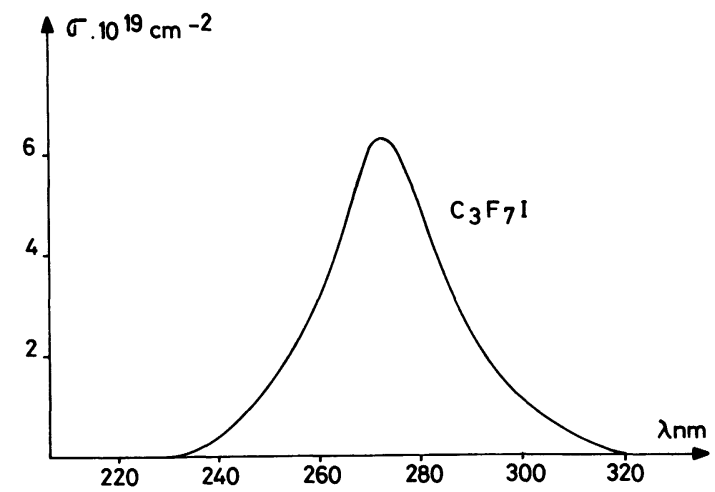

Fig. 1. - Spectre d'absorption du $\mathrm{C}_{3} \mathrm{~F}_{7} \mathrm{I}$.

$\left[\mathrm{C}_{3} \mathrm{~F}_{7} \mathrm{I}\right.$ absorption spectrum].

REVUE DE PHYSIQUE APPLIQUÉE. - T. 18, № 3, MARS 1983 que relativement simple technologiquement est assez mal connue dans les processus conduisant à l'émission ultraviolette. Une approche expérimentale en a donc été faite, pour en mieux connaître les paramètres macroscopiques. Cette étude a montré qu'une préionisation du xénon permettait d'augmenter sensiblement la puissance lumineuse et donc le rendement photonique.

2. Les flashes au xénon et pompage optique du laser à iode. - La lumière ultraviolette émise par les flashes au xénon permet par photodissociation d'un composé tel que le $\mathrm{C}_{3} \mathrm{~F}_{7} \mathrm{I}$ d'obtenir un atome d'iode excité dans l'état I $\left(2 \mathrm{P}_{1 / 2}\right)$. La grande durée de vie (quelques millisecondes) permet la création d'une inversion de population avec l'état $I\left(2 \mathrm{P}_{3 / 2}\right)$ et par là une émission stimulée à $1,315 \mu \mathrm{m}$.

Cette source UV de pompage, pour être utilisable sur des lasers de puissance à iode, doit avoir :

- un rendement photonique maximum dans la bande $250-300 \mathrm{~nm}$,

- une durée d'émission brève $(\simeq 10 \mu \mathrm{s})$ [2].

Le régime de fonctionnement des flashes est déterminé pour leur utilisation comme source de pompage d'amplificateurs laser à iode. La configuration des amplificateurs dans lesquels sont placés les flashes impose une inductance minimum $L$ au câblage. La valeur relevée lors de notre expérimentation est de $2,3 \mu \mathrm{H}$. Par ailleurs et c'est un phénomène propre au laser à iode, où des composés iodés se déposent sur la 


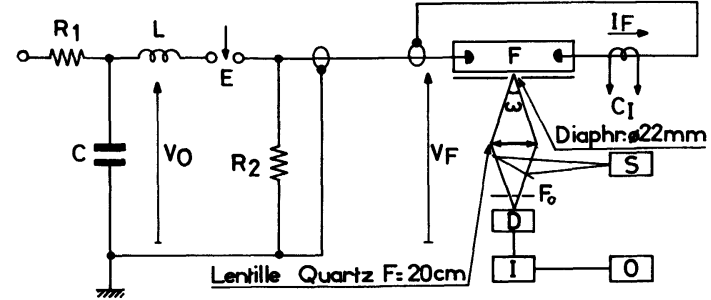

Fig. 2. - Montage expérimental utilisé pour la caractérisation des flashes au xénon : $R_{1}$ - Résistance de charge; $R_{2}$ - Résistance fixant le potentiel de l'électrode " froide " du commutateur $E$; $E$ - Commutateur d'énergie; $C-$ Capacité réservoir; $L$ - Inductance totale de la maille; $V_{0}$ - Tension maximum de charge $\mathrm{C} ; \mathrm{F}$ - Flash au xénon; $\mathrm{C}_{1}-$ Ceinture de Rogowski pour mesure $I$; $\mathrm{S}$ - Spectrographe; D - Détecteur pour mesurer le flux lumineux (photodiode silicium Edgerton type 215 B); $\mathrm{F}_{0}$ - Filtres optiques étalonnés - Filtre interférentiel centré à $2760 \AA$ de bande passante $\pm 210 \AA$ plus densités neutres étalonnées; I - Intégrateur; $\mathrm{O}$ - Oscilloscope ; $V_{\mathrm{F}}$ - Tension aux bornes du flash mesurée à l'aide d'un diviseur résistif; $I_{\mathrm{F}}-$ Courant traversant le flash mesuré à l'aide d'une ceinture de Rogowski; $\Omega$ - Angle solide d'observation.

[Experimental set up used to characterize xenon flashlamps : $R_{1}$ - Load resistance; $R_{2}$ - Resistance to fix the electrode ground voltage of switch $E ; E-$ Sparkgap; $C$ - Capacitor bank; $L-$ Total inductance of network; $V_{0}$ - Maximum voltage of $\mathrm{C} ; \mathrm{F}$ - Xenon flash-lamp; $\mathrm{C}_{\mathrm{I}}$ - Rogowski coil to measure $I_{\mathrm{F}} ; \mathrm{S}-$ Spectroscope D - Light flux detector (Edgerton silicon photodiode $215 \mathrm{~B}) ; \mathrm{F}_{0}$ - Calibrated optic filters $-2700 \AA$ filter ( $\pm 200 \AA)$ and calibrated neutral densities; I - Integrator; $\mathrm{O}$ - Oscilloscope; $V_{\mathrm{F}}-$ Flash-lamp voltage measured by resistive current divider $; I_{\mathrm{F}}-$ Current through the flash-lamp measured by a Rogowski coil; $\Omega$ - Solid angle of observation.]

face interne de l'enveloppe de quartz qui contient le milieu laser, une onde de choc centripète se crée lorsque le flux lumineux de pompage ayant traversé cette enveloppe a vaporisé une partie des composés iodés qui s'étaient déposés (Fig. 3). Cette onde de choc perturbe le milieu laser en créant des variations d'in-

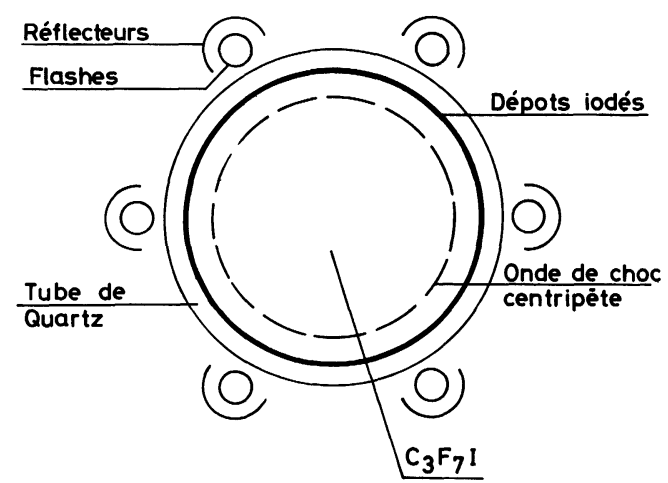

Fig. 3. - Onde de choc centripète.

[Centripetal shock wave.] dice; il faut donc éviter qu'elle ne se propage trop en avant dans ce milieu lorsque le faisceau laser va se présenter pour y être amplifié.

Comme l'inversion de population doit être voisine de son maximum lorsque l'onde laser arrive d'une part et que l'onde de choc (onde faible dont la vitesse est de l'ordre de celle du son dans le milieu considéré) ne doit pas perturber une section trop importante du faisceau d'autre part, la décharge électrique alimentant les flashes doit être rapide et engendrer une émission UV d'une durée de 10 à $15 \mu$ s (surtout dans les amplificateurs de faible diamètre).

- Une bonne fiabilité à une cadence de décharge de quelques minutes.

- Une énergie lumineuse, par unité de longueur du flash et par stéradian, élevée $\left(\simeq 4 \mathrm{~J} . \mathrm{cm}^{-1} \mathrm{sr}^{-1}\right)$.

Dans un premier temps, nous nous sommes attachés à préciser les caractéristiques des flashes utilisés jusqu'ici avec les chaînes laser, à iode, existantes.

2.1 EXPÉRIENCE. - La figure 2 précise le montage utilisé pour mesurer les paramètres photométriques et électriques des flashes. Ceux-ci sont composés d'un tube de quartz fondu de diamètre intérieur $19 \mathrm{~mm}$ et de $3 \mathrm{~mm}$ d'épaisseur. Les électrodes sont composées d'un frittage de baryum, molybdène, strontium, thorium et tungstène et la distance inter-électrode est de $1 \mathrm{~m}$. A la fabrication, le tube est rempli de 30 torrs de xénon pur et ses électrodes sont isolées, jusqu'à $60 \mathrm{kV}$, par rapport aux bagues de fixation. Nous avons utilisé des flashes fabriqués par ILC [8] et les laboratoires de Marcoussis de la C.G.E. [9]. Les mesures du flux lumineux s'effectuaient en maintenant sur la photodiode UV, D, un éclairement sensiblement constant pour conserver un même régime de fonctionnement. Pour cela des filtres neutres étalonnés étaient interposés.

2.2 Caractéristiques macrosCopiQues du PlasMA. - 2.2.1 Caractéristiques électriques et énergétiques. - Dans le montage utilisé où le condensateur

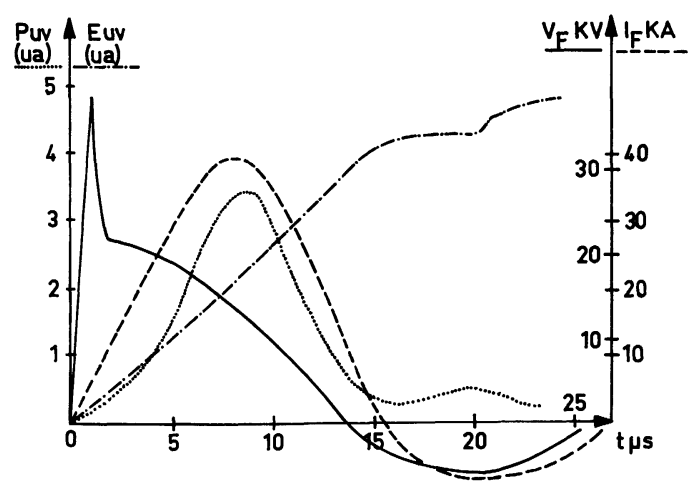

Fig. 4. - Courant dans le flash, tension aux bornes du flash, énergie lumineuse UV, en fonction du temps.

[Current through the flash-lamp with time, flash-lamp voltage with time, UV light power emitted by the flash-lamp with time.] 


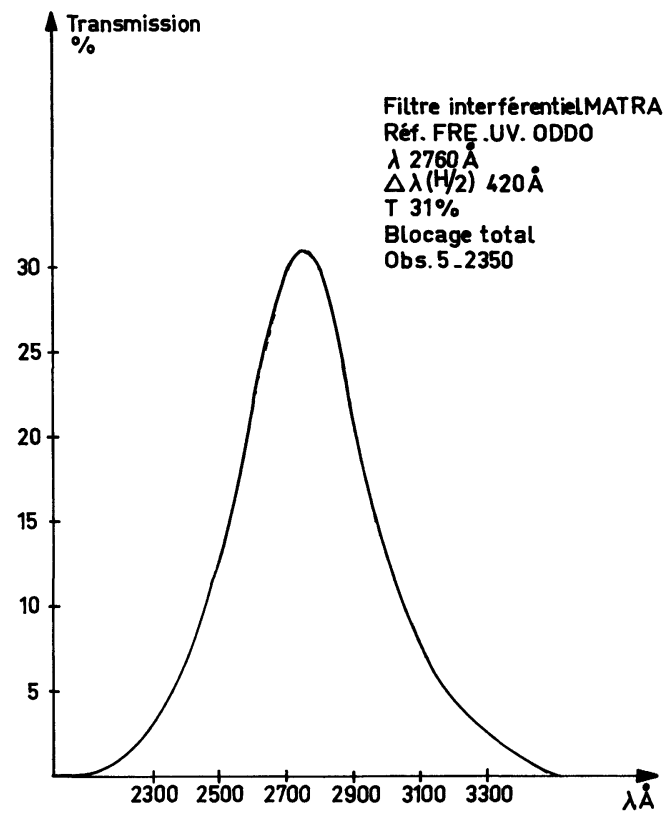

Fig. 5. - Transmission du filtre interférentiel en fonction de la longueur d'onde.

[Transmission of the interferential filter versus the wavelength.]

réservoir a une capacité de $6,24 \mu \mathrm{F}$, la self totale de la maille étant de $2,3 \mu \mathrm{H}$, la durée de l'émission lumineuse pour un régime électrique pseudo-oscillant ou amorti critique est sensiblement égal à : $t \sim \pi \sqrt{L C}=12 \mu \mathrm{s}$. Dans les études qui suivront, la maille principale aura toujours ces mêmes paramètres.

L'expérimentation débute par la recherche du maximum d'émission lumineuse UV en fonction de l'énergie électrique d'excitation du flash. Pour cela, on fait varier la tension de charge $V$ du condensateur réservoir et on relève grâce aux capteurs les valeurs de la tension aux bornes du condensateur $V_{0}$ du flash $V_{\mathrm{F}}$ et du courant $I_{\mathrm{F}}$ qui le traverse en fonction du temps (Fig. 4). L'énergie lumineuse dans le domaine UV est mesurée par une photodiode au silicium $D$ de sensibilité $180 \mathrm{~mA} / \mathrm{W}$ à $2300 \AA$ (Fig. 4), munie d'un filtre interférentiel centré à $2760 \AA$ et de bande passante $\pm 210 \AA$ (voir courbe de transmission Fig. 5).

Si on trace la courbe du rendement photonique dans l'étendue géométrique $U_{0}$ :

$$
\rho U=E_{\mathrm{UV}} / E \text { électrique stockée dans } \mathrm{C}
$$

pour différentes tensions de charge correspondant à différentes énergies stockées, on constate qu'elle passe par un maximum (Fig. 6) pour $E_{\mathrm{C}}=4,5 \mathrm{~kJ}$ correspondant à une tension de charge de $38 \mathrm{kV}$ du condensateur. Dans ces conditions, le régime de la décharge est pseudo-oscillant avec $25 \%$ d'inversion. Le courant maximum est alors de $40 \mathrm{kA}$ et il correspond à une densité de courant de $13 \mathrm{kA} / \mathrm{cm}^{2}$ dans le flash. L'impédance correspondant au maximum du courant est de $0,3 \Omega\left(V_{\mathrm{F}} / I_{\mathrm{F}}\right.$, voir Fig. 4$)$.

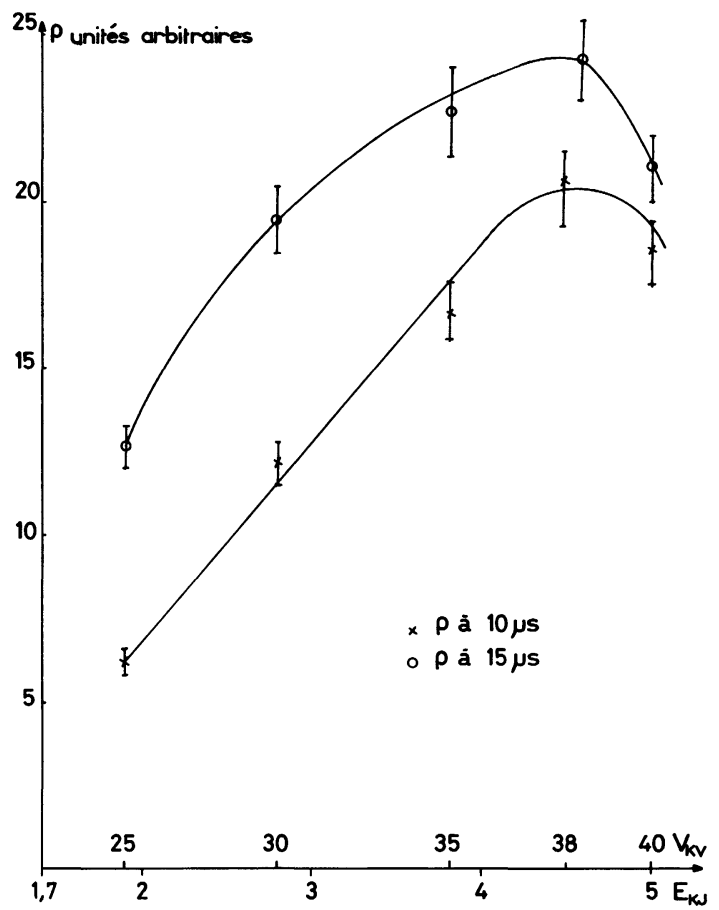

Fig. 6. - Rendement énergétique en fonction de l'énergie électrique pour $c=$ Cte.

[Energy efficiency with input capacitive energy for $c=$ Const. $]$

La précision de la mesure est d'environ $\pm 10 \%$. Compte tenu de la valeur du courant et de la section du plasma, donc de la densité de courant pour le maximum de la courbe, il est possible que la température du plasma approche $1800 \mathrm{~K}$, température optimum pour le rendement optimum à $2700 \AA$ (voir M. A. Gusinov [13]).

2.2.2 Caractéristiques spectroscopiques. - A l'aide d'un spectrographe HUET à double prisme (résolution

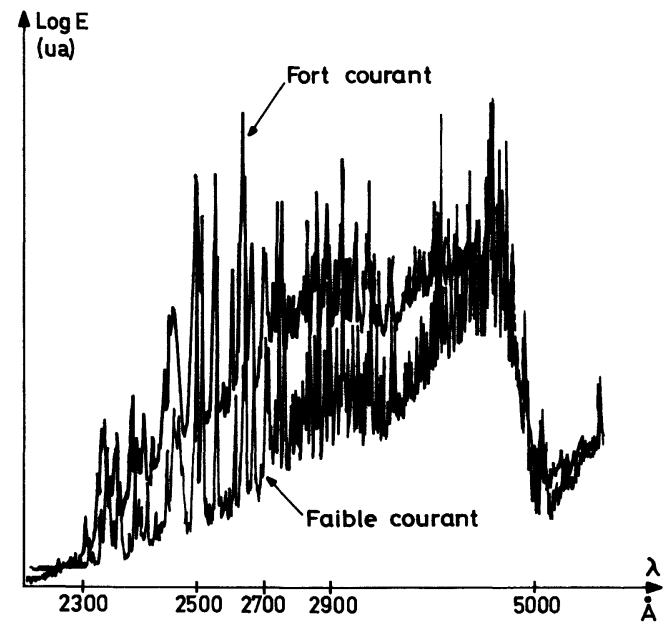

Fig. 7. - Spectre de l'émission du flash entre $220 \mathrm{~nm}$ et $680 \mathrm{~nm}$.

[Spectrum emission of the flash-lamp between $220 \mathrm{~nm}$ and $680 \mathrm{~nm}$.] 
spectrale $\simeq 7 \AA$ ), nous avons relevé le spectre d'émission du flash au xénon dans la gamme 2 200-6 $500 \AA$. La figure 7 donne le résultat obtenu. Le spectre comporte de nombreuses raies correspondant aux corps constituant les composants du flash (électrodes, tube, gaz) ou aux impuretés qu'ils peuvent contenir. Lorsqu'on augmente le courant de la décharge, le spectre se relève nettement dans la partie UV, alors que la partie visible varie peu; cela montre l'intérêt d'utiliser une décharge à haute densité de courant si l'on ne tient pas compte du rendement.

2.2.3 Caractéristiques dimensionnelles du plasma de décharge. - Nous avons étudié l'évolution spatiale et temporelle des plasmas à l'intérieur du tube flash à l'aide d'une caméra électronique TRW sensible dans l'UV et fonctionnant soit en image intégrale, soit en balayage de fente.

Régime « Image Intégrale » - Temps de pose $10 \mu \mathrm{s}$.

A l'arrière et sur une partie du flash on dispose un réflecteur cylindrique d'aluminium poli de $12 \mathrm{~cm}$ de diamètre, le tube flash étant centré au foyer de ce réflecteur. La caméra est disposée de telle façon que sur sa photocathode apparaisse une demi-image du tube flash avec son réflecteur et une demi-image sans réflecteur; il sera possible de comparer sur une même décharge l'émission lumineuse du flash avec et sans réflecteur.

Après avoir réalisé un étalonnage avec deux atténuateurs calibrés, nous avons tracé les courbes densitométriques des clichés représentés sur la figure $8 a$. Nous avons ainsi pu déterminer :

- le coefficient de réflexion du réflecteur $\sim 80 \%$;

- l'absorption par le plasma de son propre rayonnement $\sim 37 \%$. Dans le cas du laser à iode, où les flashes fonctionnent à densité de courant élevée $\left(\sim 10 \mathrm{kA} / \mathrm{cm}^{2}\right)$ et pour un rayonnement de pompage centré à $2700 \AA$, le plasma absorbe $37 \%$ de l'énergie qui le traverse. On en déduit que pour obtenir un pompage efficace, il faudra utiliser des réflecteurs dont la forme en développante de cercle, par exemple, renvoie dans le milieu laser, la lumière des flashes sans traverser le plasma de la décharge ;

- et constater que celui-ci émet plus de lumière à sa périphérie (en contact avec le tube) qu'en son centre (Fig. 9a).

Régime « Balayage de Fente » - Durée $20 \mu$ s.

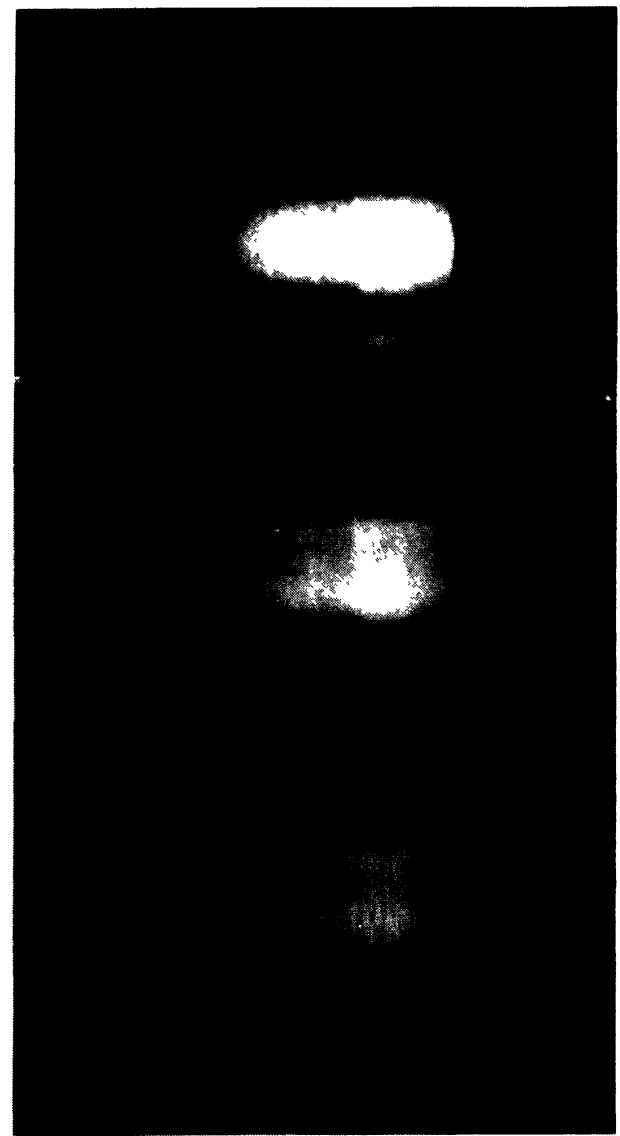

TUBE FLASH ILC $\varnothing 19-\mathrm{L} 1 \mathrm{~m}-\mathrm{P} 30 \mathrm{~T}$

IMAGE INTÉGRALE TEMPS DE POSE : $10 \mu \mathrm{s}$

LUMIÈRE UV : $2700 \AA$

b) $40 \mathrm{kV}$ avec préionisation de $40 \mathrm{~ms}$.

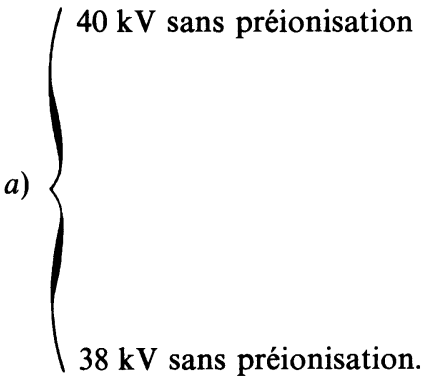

Fig. 8. - Photographie en image intégrale (temps de pose $10 \mu \mathrm{s}$ ) de la lumière UV émise par le flash avec réflecteur partiel : a) $40 \mathrm{kV}$ et $38 \mathrm{kV}$ sans préionisation, $b$ ) $40 \mathrm{kV}$ avec préionisation.

[Integral image photography (time of exposure $10 \mu \mathrm{s}$ ) of UV light emitted by the flash-lamp with partial reflector :a) $40 \mathrm{kV}$ and $38 \mathrm{kV}$ without pre-ionization, b) $40 \mathrm{kV}$ with pre-ionization.] 


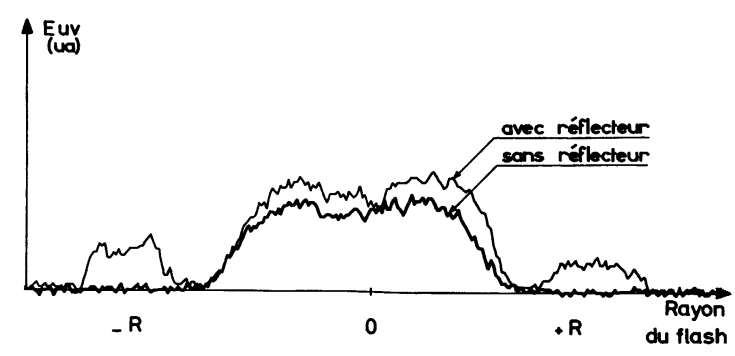

Fig. 9a. - Densitométrie en fonction du rayon sans préionisation.

[Light density as a function of radius without pre-ionization.]

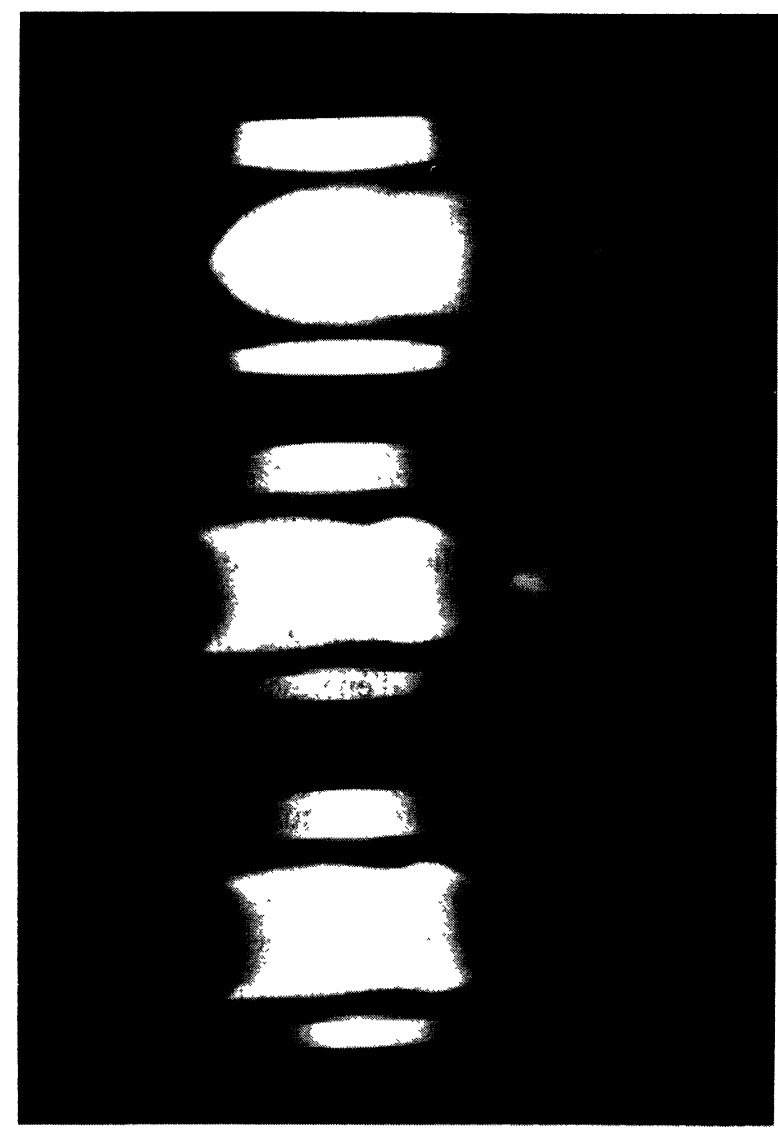

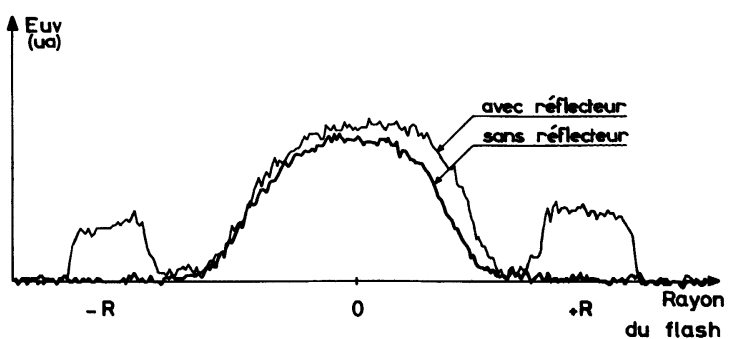

Fig. $9 b$. - Densitométrie en fonction du rayon avec préionisation.

[Light density as a function of radius with pre-ionization.]

TUBE FLASH ILC $\varnothing 19-\mathrm{L} 1 \mathrm{~m}-\mathrm{P} 30 \mathrm{~T}$

BALAYAGE DE FENTE $\mathrm{T}: 20 \mu \mathrm{s}(46 \mathrm{~mm})$

LUMIÈRE UV : $2700 \AA$

b) $40 \mathrm{kV}$ avec préionisation de $40 \mathrm{~ms}$.

a)

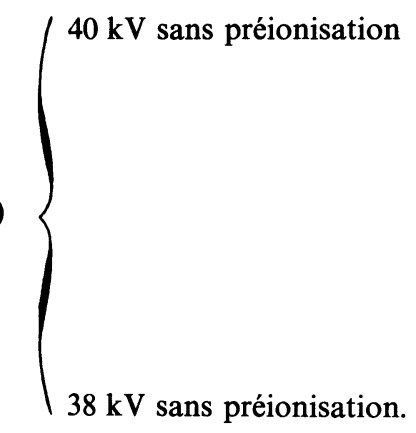

Fig. 10. - Photographie en balayage de fente $(t=20 \mu \mathrm{s})$ de la lumière $\mathrm{UV}: \dot{a}) 40 \mathrm{kV}$ et $38 \mathrm{kV}$ sans préionisation, $b) 40 \mathrm{kV}$ avec préionisation.

[Steak camera photography (split aperture $t=20 \mu \mathrm{s}$ ) of UV light emitted by a flash-lamp with reflector :a) $40 \mathrm{kV}$ and $30 \mathrm{kV}$ without pre-ionization, b) $40 \mathrm{kV}$ with pre-ionization.]

Nous réalisons sur la photocathode de la caméra l'image d'une fente de $0,2 \mathrm{~mm}$ de large placée devant le tube flash muni de son réflecteur.

Sur la figure $10 a$, on constate que le plasma naît sur la surface interne du tube et évolue sous forme d'un ruban.

A partir des clichés, en traçant les courbes isodensitométriques (Fig. 11) et en leur attribuant des valeurs propres tenant compte de l'étalonnage précédent, on peut représenter le profil spatial de l'émission lumineuse (Fig. 12).
2. 3 EVAlUation DU RENDEMENT PHOTONIQUE DES FLASHES. - Dans le paragraphe précédent, nous avons déterminé les conditions d'alimentation électrique du flash qui conduisaient au meilleur rendement relatif de l'émission UV, nous avons alors tracé l'indicatrice de cette émission dans le plan de l'axe du flash et dans un plan de section droite. La forme de ces indicatrices montre qu'avec une bonne approximation, l'émission se fait suivant la loi de Lambert, nous pouvons donc écrire :

$$
L_{\mathrm{e}}=\frac{\phi_{\mathrm{e}}}{\Omega S_{\mathrm{e}}},
$$




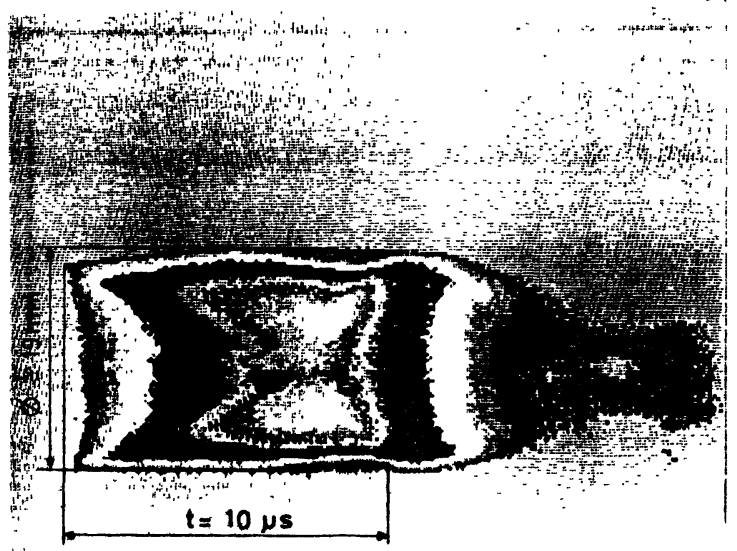

Fig. 11. - Densitométrie intégrale de la photographie $10 a$ à $38 \mathrm{kV}$.

[Integral light density of the photography $10 a$ at $38 \mathrm{kV}$.]

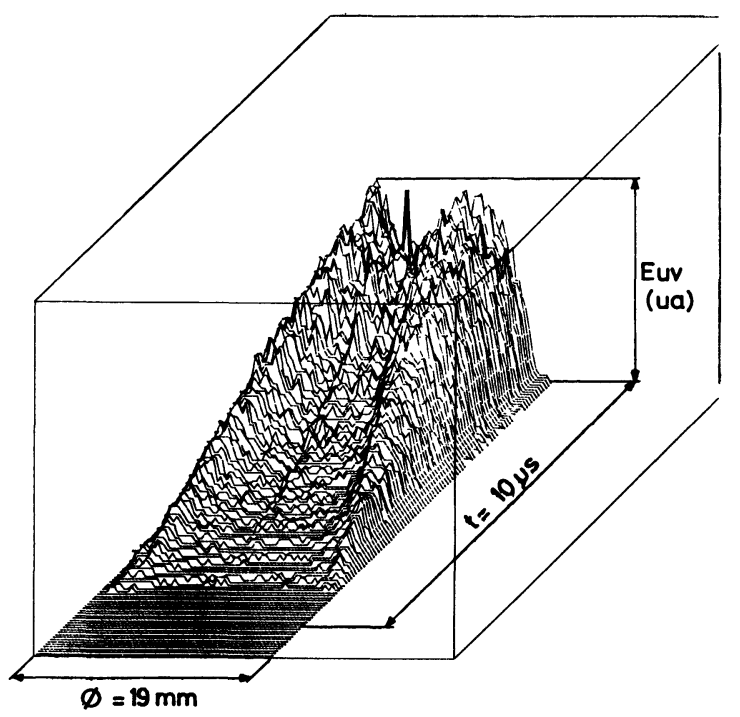

Fig. 12. - Profil spatial de l'émission lumineuse sans préionisation.

[Spatial profile of light emission without pre-ionization.]

$L_{\mathrm{e}}$ : luminance énergétique,

$\phi_{\mathrm{e}}$ : flux énergétique,

$\Omega$ : angle solide sous lequel le détecteur voit un point du plasma,

$S_{\mathrm{e}}$ : surface émettrice.

Par ailleurs, le flux énergétique total $\phi_{\mathrm{t}}$ émis par le flash s'exprime par :

$$
\phi_{\mathrm{t}}=L_{\mathrm{e}} \cdot \pi \cdot S_{\mathrm{t}}
$$

avec $S_{\mathrm{t}}$ surface totale émettrice.

Si $\rho$ est le rendement du flash dans la bande $2700 \pm$ $200 \AA$, il a pour expression :

$$
\rho=\frac{\phi_{\mathrm{t}}}{\phi_{\mathrm{F}}}
$$

$\phi_{\mathrm{F}}$ étant l'énergie électrique stockée dans le condensateur réservoir.

Pour une énergie électrique stockée de $4,5 \mathrm{~kJ}$, on obtient un rendement de $(1,62 \pm 0,67) \%$; il se décompose de la façon suivante :

$\mathrm{Si}$

$E_{\mathrm{eC}}=$ énergie électrique stockée dans le banc de condensateurs (connu à $\pm 10 \%$ près),

$E_{\mathrm{eF}}=$ énergie électrique aux bornes des flashes.

$E_{\mathrm{s}}=$ énergie lumineuse totale rayonnée dans tout le spectre et mesurée à l'aide d'un calorimètre.

$E_{\mathrm{UV}}=$ énergie UV émise dans la bande spectrale utile (2 $700 \pm 200) \AA ̊$ pendant les 10 premières microsecondes.

On mesure :

$\rho_{\mathrm{T}}=$ rendement de transmission électrique : $E_{\mathrm{eF}} / E_{\mathrm{eC}}=0,9 \pm 0,1$;

$\rho_{\mathrm{P}}=$ rendement d'émission du plasma $: E_{\mathrm{s}} / E_{\mathrm{eF}}=$ $0,4 \pm 0,04$

$\rho_{\mathrm{UV}}=$ rendement de l'émission ultraviolette : $E_{\mathrm{Uv}} / E_{\mathrm{s}}=0,045 \pm 0,009$;

soit un rendement total $\rho=\rho_{\mathrm{T}} \times \rho_{\mathrm{P}} \times \rho_{\mathrm{UV}} \simeq$ $(1,62 \pm 0,67) \%$.

Cette valeur prend en compte l'ensemble des pertes possibles et un temps d'observation proche de ceux utilisés dans les amplificateurs à iode.

3. Effet d'une préionisation des flashes. - Pour accroître la production d'énergie UV vers $2700 \AA$, plusieurs procédés sont utilisables :

- dopage du gaz [10],

- préionisation haute fréquence (effet "surfatron ") [11],

- préionisation par décharge à faible énergie («Simmer Courant » ou " Double Décharge ») [12].

C'est ce dernier procédé que nous avons étudié et nous en comparons les résultats avec ceux obtenus avec une décharge simple présentée dans le paragraphe précédent.

3.1 CirCuIT DE DÉCHARGE. - Il est présenté sur la figure 13.

La préionisation consiste à établir une décharge à faible énergie dans le tube flash avant que ne soit établie la décharge principale dans les conditions nominales de fonctionnement. Cette préionisation peut être obtenue à l'aide de capacité réservoir indépendante délivrant l'énergie $E_{\mathrm{p}}$ pendant le temps $t_{\mathrm{p}}$ où en prélevant une partie de l'énergie de la capacité réservoir de la maille de décharge principale ; c'est cette seconde voie que nous avons développée, car elle simplifie la réalisation pratique du dispositif.

Pour optimiser les paramètres de préionisation qui rendent maximum l'émission UV du flash, nous avons étudié l'influence de la durée et de l'intensité de la décharge de préionisation. Pour un tube flash de $1 \mathrm{~m}$ de long et de $19 \mathrm{~mm}$ de diamètre intérieur, un optimum 


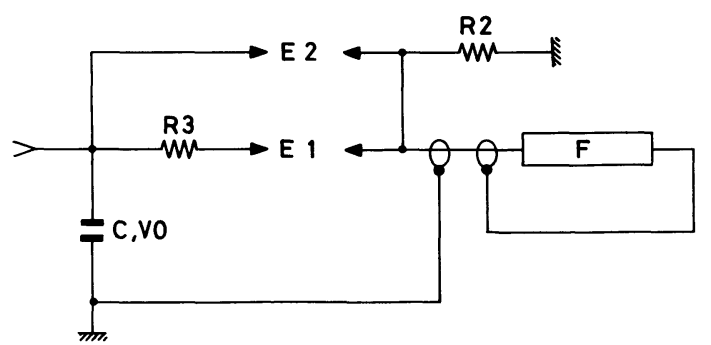

Fig. 13. - Schéma de principe du circuit permettant la préionisation.

[Principle diagram of pre-ionization time measurements.]

apparaît pour un courant de $400 \mathrm{~mA}$ appliqué durant $40 \mathrm{~ms}$. La figure 14 montre la variation relative de l'énergie UV émise par le flash en fonction de la durée d'application d'un courant de préionisation de $400 \mathrm{~mA}$.

Pour obtenir ces conditions, le condensateur réservoir est chargé à une tension $U_{0}$ de $40 \mathrm{kV}$ et stocke une énergie de $5 \mathrm{~kJ}$, après la pré-décharge de $40 \mathrm{~ms}$, la décharge principale s'amorce sous $38 \mathrm{kV}$ pour une énergie de 4,5 kJ.

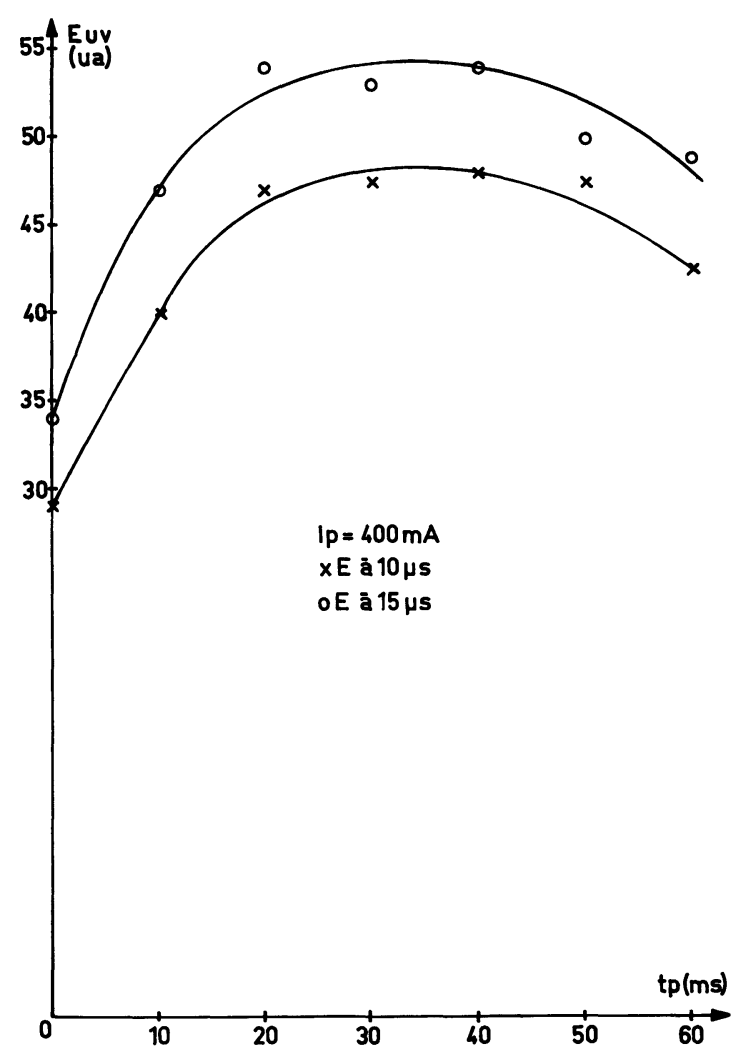

Fig. 14. - Energie UV émise en fonction du temps de préionisation.

[UV energy emitted with pre-ionization time.]

3.2 EFFETS DE LA PRÉIONISATION SUR LES CARACTÉRISTIQUES ÉLECTRIQUES DE LA DÉCHARGE. - Courant. - La variation temporelle du courant est sensiblement égale avec ou sans préionisation. On notera toutefois qu'avec préionisation, le temps de montée est légèrement plus court et l'amplitude un peu supérieure.

Tension. - A l'instant de la fermeture du commutateur de préionisation $E_{1}$, la tension aux bornes du flash est de $40 \mathrm{kV}$, après son amorçage cette tension se stabilise à environ $1000 \mathrm{~V}$ pendant $40 \mathrm{~ms}$, ce qui correspond à une impédance de $2500 \Omega$, le courant étant de 0,4 A. Lorsque le commutateur de la décharge principale $E_{2}$ se ferme à son tour, une tension de $38 \mathrm{kV}$ apparaît aux bornes du flash dont l'impédance est encore de $2500 \Omega$ (contrairement à une valeur infinie en l'absence de préionisation).

3.3 EFFET DE LA PRÉIONISATION SUR LES CARACTÉRISTIQUES SPECTROSCOPIQUES. - Dans la bande 2500 $2900 \AA$, les spectres enregistrés avec et sans préionisation sont identiques et sensiblement homothétiques. Il n'y a ni apparition ni disparition de raies, mais leurs amplitudes et celle du continuum sont relevées d'environ $2 \mathrm{~dB}$, ce qui correspond à un accroissement d'émission lumineuse d'environ $60 \%$. On notera que ces essais ont été effectués, dans les deux cas, en l'absence de réflecteur.

3.4 EFFET DE LA PRÉIONISATION SUR L'ÉMISSION D'ÉNERGIE UV. - Pour étudier la valeur de l'énergie émise dans la gamme UV autour de $2700 \AA$ et sa variation temporelle, nous utilisons le dispositif qui a déjà été décrit au paragraphe 2.2.1.

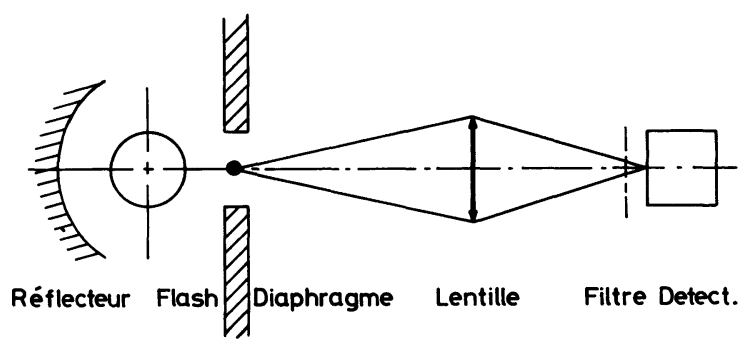

Fig. 15. - Montage expérimental permettant la mesure globale de la lumière émise.

[Experimental set up to measure of emitted light.]

Les figures $16 a$ et $16 b$ présentent les mesures effectuées et leur dépouillement avec et sans préionisation. Lorsqu'on effectue une préionisation, le temps de montée de la courbe d'énergie diminue et l'amplitude du maximum augmente, on constate dans tous les cas qu'on obtient un accroissement de l'énergie UV émise et du rendement de la décharge. Cet accroissement du rendement est évalué en tenant compte de l'énergie nécessaire à la préionisation. Deux méthodes ont été utilisées :

- méthode globale, elle intègre toute la lumière émise par une tranche complète du tube flash et reçue par le détecteur (Fig. 15),

- méthode d'analyse ponctuelle, semblable à la précédente, elle utilise deux diaphragmes de $2 \mathrm{~mm}$ de 


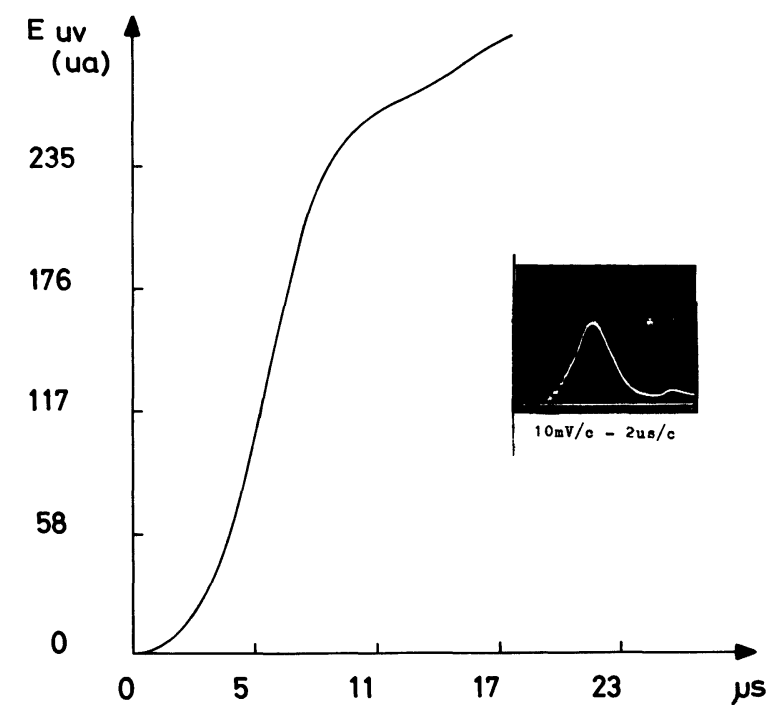

Fig. 16a. - Puissance et énergie lumineuse en fonction du temps sans préionisation.

[Light power and light energy with time without preionization.]

diamètre distants de $41 \mathrm{~cm}$, suivis d'une cellule et de ses filtres associés. Le dispositif de mesure est déplacé depuis le centre géométrique du tube flash jusqu'à l'extérieur, ce qui permet d'analyser la fraction de l'énergie lumineuse renvoyée par le réflecteur. Cette seconde méthode a permis d'obtenir les courbes comparatives de la figure 17, tracées en unités arbitraires.

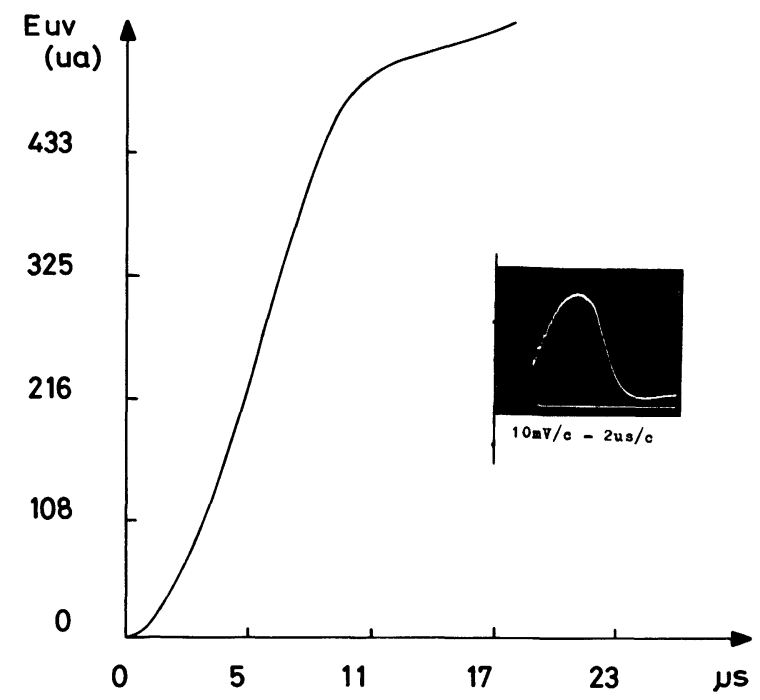

Fig. 16b. - Puissance et énergie lumineuse en fonction du temps avec préionisation.

[Light power and light energy with time with pre-ionization.]

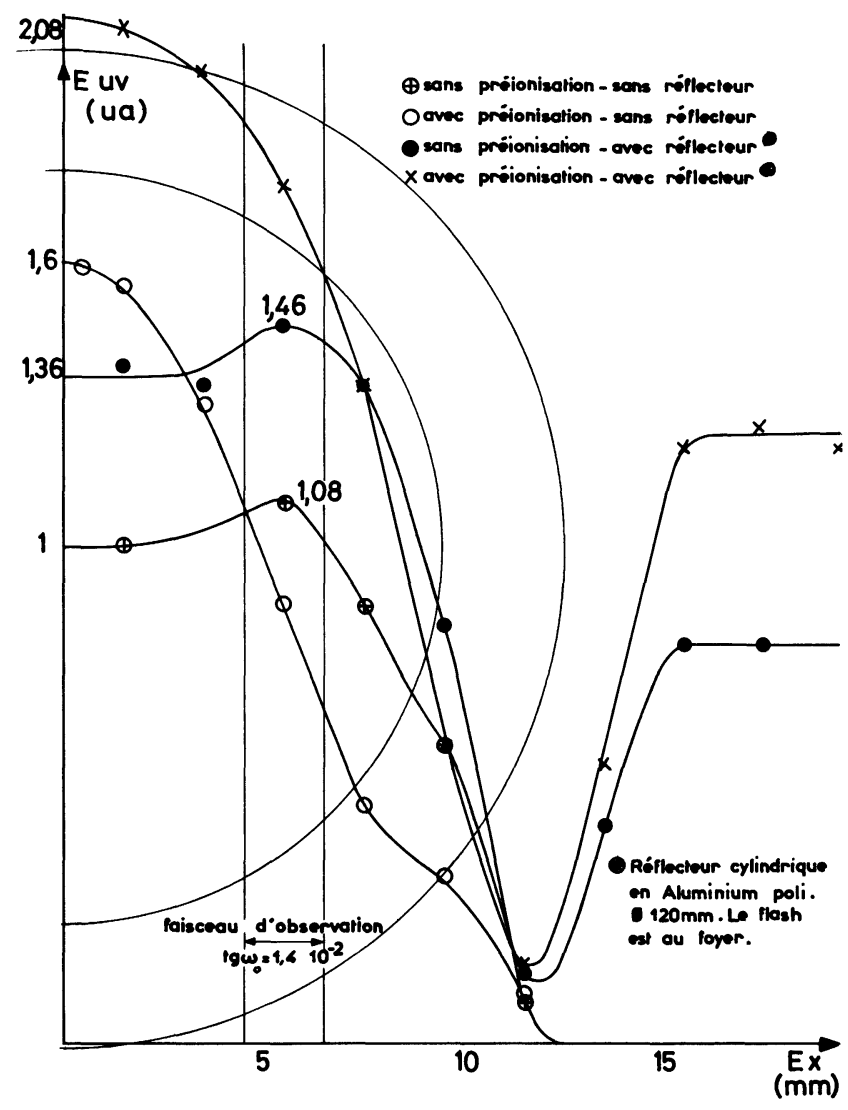

Fig. 17. - Energie lumineuse en fonction du rayon du flash mesurée par la méthode globale.

[Integrated light energy as a function of flash-lamp radius.]

Le tableau I résume les résultats obtenus avec les 2 méthodes :

Tableau I. - Energie relative lumineuse émise dans la bande spectrale UV pendant un fonctionnement de $10 \mu \mathrm{s}$ des flashes.

\begin{tabular}{|c|c|c|c|c|}
\hline & \multicolumn{2}{|c|}{ Sans réflecteur } & \multicolumn{2}{|c|}{ Avec réflecteur } \\
\hline & Centre & Global & Centre & Global \\
\hline Sans préionisation & $E_{\mathrm{C}}$ & $E_{\mathrm{G}}$ & $1,35 E_{\mathrm{C}}$ & $1,4 E_{\mathrm{G}}$ \\
\hline Avec préionisation & $1,6 E_{\mathrm{C}}$ & $1,55 E_{\mathrm{G}}$ & $2,1 \quad E_{\mathrm{C}}$ & $2,2 E_{\mathrm{G}}$ \\
\hline
\end{tabular}

En présence de préionisation, l'émission du plasma autour de $2700 \AA$ au centre du tube flash est supérieure à celle obtenue aux bords, alors que c'est l'inverse qui se produit en l'absence de préionisation; ceci est confirmé par le dépouillement densitométrique des clichés obtenus à l'aide d'une caméra électronique :

- en image intégrale, avec un temps de pose de $10 \mu$ s (Fig. 9b),

- en balayage de fente (Fig. 10b). Dans ce cas, on constate que le plasma se crée au centre du tube flash 
et s'étend vers les bords du tube à une vitesse de $2 \mathrm{~mm} / \mu \mathrm{s}$.

Les figures 13 et 19 donnent respectivement les courbes isudensitométriques et la répartition spatiale de l'énergie lumineuse que l'on peut extraire du cliché obtenu par balayage de fente ; on comparera utilement ces deux figures avec leurs homologues 11 et 12 obtenues en l'absence de préionisation.

3. 5 EFFETS DE LA PRÉIONISATION SUR UN AMPLIFICATEUR DE $70 \mathrm{~mm}$. - Les travaux de laboratoire exposés au paragraphe précédent ont confirmé que l'émission d'un tube flash, dans une gamme de longueurs d'ondes centrée sur $2700 \AA$ augmentait notablement, si on préionisait le gaz du flash avant d'y appliquer la décharge principale. De tels flashes étant utilisés pour pomper les amplificateurs du laser à iode, nous avons expérimenté les effets de la préionisation des flashes sur un amplificateur de $70 \mathrm{~mm}$ de diamètre utilisé sur une chaîne laser à iode implantée aux laboratoires de Marcoussis de la C.G.E.

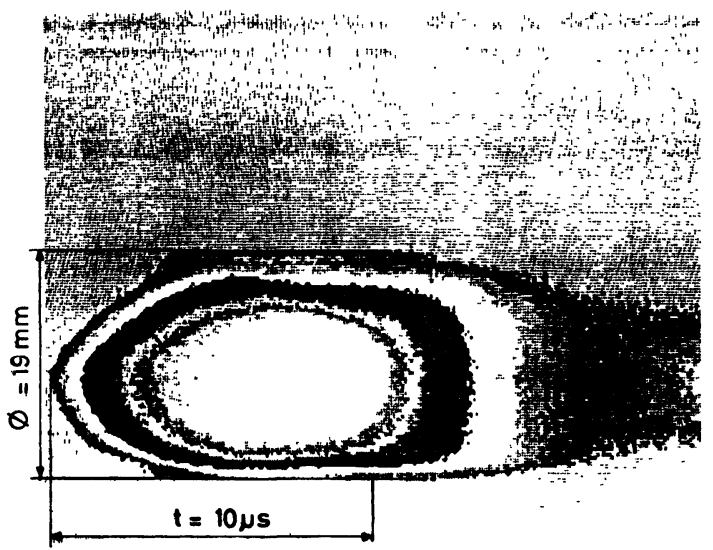

Fig. 18. - Densitométrie intégrale de la photographie $10 b$.

[Densitometry profile of the photography $10 \mathrm{~b}$.]

Cet amplificateur de $70 \mathrm{~mm}$ de diamètre utile est normalement pompé par 8 flashes de $1 \mathrm{~m}$ de long et de $19 \mathrm{~mm}$ de diamètre intérieur, identiques à ceux précédemment testés. Chaque tube flash est alimenté par la décharge d'un condensateur de 7,5 $\mu \mathrm{F}$ chargé au maximum à $35 \mathrm{kV}$. La préionisation est obtenue par une boucle indépendante du circuit de puissance (condensateur de $22,5 \mu \mathrm{F}$ chargé au maximum à $30 \mathrm{kV}$ ), cette boucle est commune aux 8 flashes. Avec ce dispositif, le courant de préionisation diminue de $20 \%$ pendant les $40 \mathrm{~ms}$ de son application, alors qu'il ne variait que de $5 \%$ dans le montage expérimental décrit dans les paragraphes précédents.

On détermine la quantité d'énergie émise en oscillateur par l'amplificateur placé entre 2 miroirs-plans (100\% et $30 \%$ de coefficients de réflexion respectifs) pour une pression du composé iode $\mathrm{C}_{3} \mathrm{~F}_{7} \mathrm{I}$ de 8 torrs et une pression d'argon de 200 torrs. La figure 20 présente les résultats obtenus en fonction de l'énergie

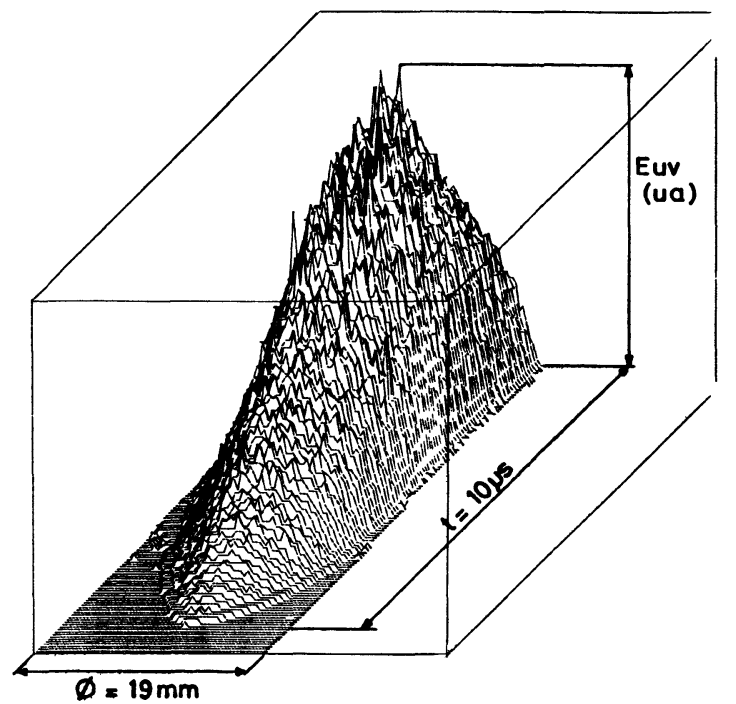

Fig. 19. - Profil spatial de l'émission lumineuse avec préionisation.

[Spatial profile of light emission with pre-ionization.]

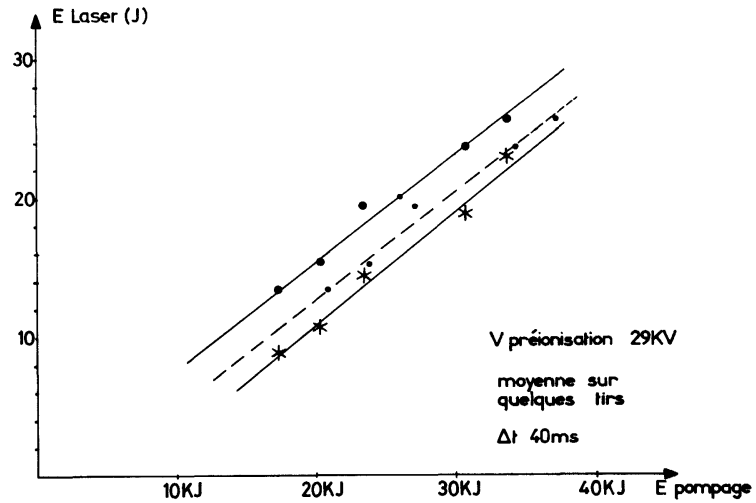

Fig. 20. - Energie laser en fonction du pompage.

[Laser energy as a function of the capacitors-bank energy.]

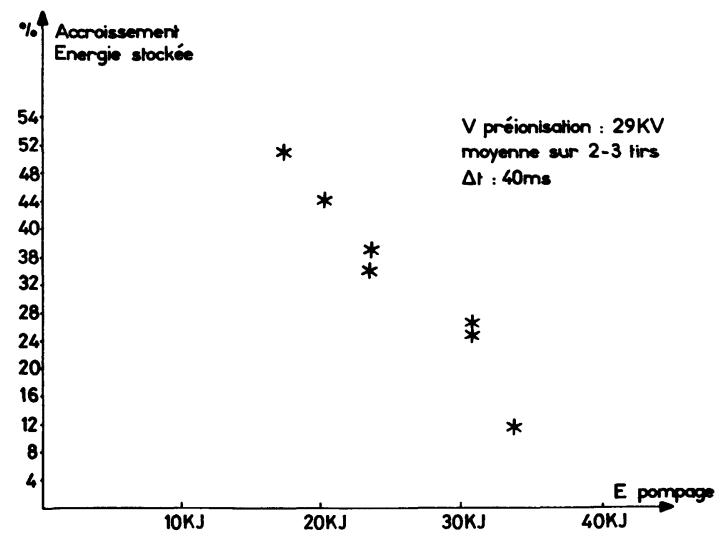

Fig. 21. - Accroissement de l'énergie stockée en fonction du pompage.

[Increase of laser storage energy as a function of the capacitors-bank energy.] 
totale de pompage pour les huit flashes et pour une tension de préionisation de $29 \mathrm{kV}$. La figure 21 indique les valeurs de l'accroissement du rendement de pompage, en fonction de l'énergie délivrée aux flashes. A faible niveau ( $2 \mathrm{~kJ}$ par flash), l'amélioration est de $60 \%$, mais elle tombe à $20 \%$ pour l'énergie nominale de $4,5 \mathrm{~kJ}$ par flash.

4. Commentaires et conclusion. - Dans un article [13], M. A. Gusinow indique que la température électronique du plasma qui remplit l'intérieur d'un tube flash lors de la décharge peut s'exprimer par la relation empirique :

$$
T=603\left(J \cdot R^{1 / 2}\right)^{4 / 11}
$$

où

$T$ est la température du plasma en degrés Kelvin, $J$ est la densité de courant en $\mathrm{A} / \mathrm{cm}^{2}$,

$R$ est le diamètre du plasma en $\mathrm{cm}$.

Appliqué au cas d'un tube flash de $19 \mathrm{~mm}$ de diamètre intérieur soumis à une décharge produisant une densité de courant uniforme de $13000 \mathrm{~A} / \mathrm{cm}^{2}$, la relation précédente conduit à une température de plasma de $18780 \mathrm{~K}$. Or M. A. Gusinov montre que le rendement d'émission dans la bande 2 500-2 $900 \AA$ est maximum pour une température de plasma de $18000 \mathrm{~K}$.

En l'absence de préionisation, le plasma de la décharge ne remplit pas complètement le tube ainsi que le montrent les enregistrements cinématographiques, la densité locale de courant s'accroît donc, ce qui ne permet pas d'atteindre le maximum de rendement d'émission ultraviolette.

Outre l'accroissement de l'émission UV, donc celui du rendement de pompage du laser à iode, l'application de la préionisation permet :

- d'améliorer la fiabilité des flashes par suite de la diminution de la densité du courant et de son homogénéisation dans tout le volume du tube flash,

- d'augmenter notablement la quantité de lumière UV renvoyée par les réflecteurs :

- à travers le tube flash, car le rayonnement UV traverse un plasma moins chaud donc moins absorbant,

- autour du tube, car le plasma étant moins turbulent et plus homogène, une forme appropriée des réflecteurs permet d'en accroître l'efficacité,

- d'autoriser la décharge principale seulement si l'information de la préionisation est correcte c'est-àdire caractéristique du bon état du flash.

Nos expériences ont donc montré que la préionisation améliorait le rendement de l'émission UV

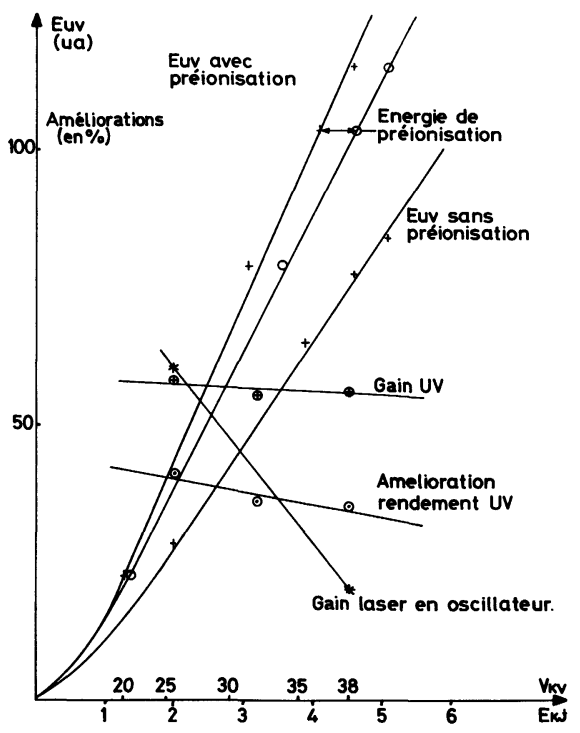

Fig. 22. - Energie, gain UV et gain laser

[UV energy, UV improvement and laser storage energy.]

des flashes au xénon utilisés pour le pompage du laser à iode. Toutefois, nous avons noté que l'accroissement relatif de l'émission UV des flashes $\Delta E_{\mathrm{UV}} / E_{\mathrm{UV}}$ passait de $60 \%$ à $45 \%$ environ lorsque l'énergie électrique de pompage variait de 2 à $4,5 \mathrm{~kJ} /$ flash, alors que l'émission laser elle-même subissait un accroissement relatif $\Delta E_{1} / E_{1}$ passant de $60 \%$ à $20 \%$ dans les mêmes conditions.

La figure 22 résume ces résultats que l'on peut expliquer comme suit :

- le terme $\Delta E_{\mathrm{UV}} / E_{\mathrm{Uv}}$ a été mesuré à $10 \mu \mathrm{s} d u$ déclenchement des flashes c'est-à-dire au moment où l'impulsion incidente se présente à l'amplification,

- le terme $\Delta E_{1} / E_{1}$ déterminé sur l'amplificateur utilisé en oscillateur est mesuré pour la durée totale de l'impulsion UV de pompage soit environ $20 \mu \mathrm{s}$,

- l'auto-absorption du plasma des flashes à son propre rayonnement UV s'accroît au fur et à mesure que la densité du courant de décharge augmente,

- il faut noter que ces résultats ont été obtenus avec des lampes flashes bien particulières employées sur des amplificateurs de puissance de laser à iode (longueur inter-électrodes : $1 \mathrm{~m}$; diamètre intérieur : $19 \mathrm{~mm}$; pression de xénon : 30 torrs). Nous pensons que ces résultats et nos conclusions ne sont pas valables pour d'autres flashes.

Remerciements. - Nous tenons à remercier messieurs Husson et Poussier pour leur assistance technique ainsi que messieurs Farcy et Beaupère des laboratoires de Marcoussis (C.G.E.) pour les essais qu'ils ont effectués sur l'amplificateur à iode $\varnothing 70 \mathrm{~mm}$. 


\section{Bibliographie}

[1] Kasper, J. V. et Pimentel, C. G., Appl. Phys. Lett. 5 No 11 (1964) p. 231.

[2] Friart, D., Rapport CEA - R 5014/Octobre 1979.

[3] Guyot, J. C., Farcy, J. C. et Guillet, H., Rev. Phys. Appl. 12 (1977) 1789.

[4] Basov, N. G., Zuev, V. S., Il Nuovo Cimento 31 No 1.

[5] Klement, G. et WedleR, H., Rapport Interne IPP Garching.

[6] Moffat, J. et Maynard, R., Rapport ILC-Technology, No 7410, 1er novembre 1974.

[7] Farcy, J. C., Huguet, M., Pugliese, V. et Goin, C., Topical Meeting on International Confinement Fusion, 26-28 février 1980.
[8] Lovoi, P. et Maynard, R., Rapport ILC Technology, No 782, mars 1978.

[9] FarCy, J. C., Communication privée.

[10] Palmer, R. E. et Palmer, M. A., Rapport Sandia, 782205 de janvier 1979.

[11] PoueY, M. et LePrince, P., Rapport de Laboratoire de Physique des Plasmas (Paris-Sud) Orsay, novembre 1979.

[12] Емmett, J. L. et Schalow, A. L., Appl. Phys. Lett. 2 (1963) 204-206.

[13] Gusinow, M. A., IEEE Quantum Electron. 12 (1975) p. 929-954. 